\title{
Riesgo suicida en estudiantes universitarios de Manizales (Caldas, Colombia) ${ }^{5}$
}

\author{
José Jaime Castaño Castrillón* \\ Magister Scientiae en Ciencias Físicas \\ Universidad de Manizales, Caldas, Colombia \\ Correo electrónico: jcast@umanizales.edu.co

\section{Sandra Constanza Cañón Buitrago} \\ PhD Ciencias Sociales Niñez y Juventud \\ Universidad de Manizales, Caldas, Colombia \\ Correo electrónico: sandraka@umanizales.edu.co \\ Juan José López Tamayo \\ Pregrado en Medicina \\ Universidad de Manizales, Caldas, Colombia \\ Correo electrónico: julopeztama@hotmail.com
}

Recibido: 05/02/2020

Evaluado: 02/06/2020

Aceptado: 24/06/2020

\section{Resumen}

Se pretende determinar el factor de riesgo suicida y factores asociados en población de estudiantes universitarios de una universidad de Manizales (Colombia). Se trata de un estudio de corte transversal. Se escogieron 170 estudiantes mediante un muestreo por conveniencia aplicando las siguientes escalas: Cuestionario IS0-30, APGAR, Inventario de Beck, Escala de Rosenberg, Cuestionario Scoff, Escala AUDIT y Escala Epworth. El 19.4\% de los participantes se situó en el nivel alto de riesgo suicida, siendo de género femenino el $55.9 \%$ de estos. Las siguientes variables resultaron significativas en el análisis bivariado: género, consumo de sustancias psicoactivas, funcionalidad familiar, depresión, autoestima, dependencia bebidas alcohólicas y somnolencia. En el análisis multivariado resultaron significativas funcionalidad familiar y autoestima. Es una población con alto riesgo suicida por la presencia de factores de riesgo, lo cual requiere intervención urgente, no solo en esta población, sino también en la población universitaria de Manizales.

Palabras clave

Factores de riesgo, suicidio, estudiantes, conductas de riesgo para la salud.

5 Para citar este artículo: Castaño-Castrillón, J.J., Cañón-Buitrago, S.C. y López-Tamayo, J.J. (2022). Riesgo suicida en estudiantes universitarios de Manizales (Caldas, Colombia). Informes Psicológicos, 22(1), pp. 77-95 http://dx.doi.org/10.18566/infpsic.v22n1a05

* QEPD 


\section{Suicidal risk in college students from Manizales (Caldas, Colombia)}

\section{Abstract}

This cross-sectional study seeks to determine the suicide risk factor and associated factors in college students from a university located in Manizales (Colombia). 170 students were chosen by convenience sampling applying the following scales: ISO-30 Questionnaire, APGAR, Beck Inventory, Rosenberg Scale, Scoff Questionnaire, AUDIT Scale and Epworth Scale. 19.4\% of the participants were at a high level of suicide risk, $55.9 \%$ of them being female. The following variables were significant in the bivariate analysis: gender, consumption of psychoactive substances, family functionality, depression, self-esteem, dependence on alcoholic beverages, and sleepiness. In the multivariate analysis, family functionality and self-esteem were significant. This population has a high suicide risk due to the presence of risk factors, which requires urgent intervention, not only in this population, but also in the university population of Manizales.

Keywords

Risk factors, suicide, students, health risk behaviors.

\section{Risco de suicídio em estudantes universitários de Manizales (Caldas, Colômbia) \\ Resumo}

Pretende-se determinar o fator de risco de suicídio e fatores associados em uma população de estudantes universitários de Manizales (Colômbia). Trata-se de um estudo transversal no qual 170 alunos foram escolhidos por amostragem de conveniência aplicando-se as seguintes escalas: Questionário IS0-30, APGAR, Inventário de Beck, Escala de Rosenberg, Questionário de Scoff, Escala AUDIT e Escala de Epworth. 19,4\% dos participantes apresentavam alto risco de suicídio, sendo $55,9 \%$ do sexo feminino. As seguintes variáveis foram significativas na análise bivariada: sexo, consumo de substâncias psicoativas, funcionalidade familiar, depressão, autoestima, dependência de bebidas alcoólicas e sonolência. Na análise multivariada, as variáveis funcionalidade familiar e autoestima foram significativas. Determinou-se que se trata de uma população com alto risco de suicídio devido à presença de fatores de risco, o que requer intervenção urgente, não só na população amostral, mas também na população universitária de Manizales.

Palavras chave

Fatores de risco, suicídio, estudantes, comportamentos de risco à saúde. 


\section{ntroducción}

El suicidio y los comportamientos suicidas se han convertido en un problema de salud pública cuya atención debe ser multidisciplinaria y multisectorial (Micin \& Bagladi, 2011). Entidades como la Organización Mundial de la Salud (2019) y la Organización Panamericana de la Salud (2018) consideran que el suicidio es la tercera causa de muerte en personas menores de 45 años. En estas etapas de la vida surgen dificultades para afrontar y resolver los estresores, y problemas para regular y controlar las emociones (Ceballos \& Suárez, 2012).

En el citado proceso aparecen múltiples manifestaciones, entre ellas, la ideación suicida, entendida como las ideas o pensamientos reiterativos que se generan en un individuo sobre la muerte (Sánchez-Sosa, Villareal-González, Musitu, \& Martínez-Ferrer, 2010). Es la fase inicial de un proceso cuyo fin último es el suicidio, por lo tanto, se debe considerar como la fase más importante para prevenir el acto suicida. Se estima que el suicidio se ubica entre las primeras cinco causas de muerte en jóvenes con edades entre los 15 y 19 años (Córdova-Osnaya, Rosales-Murillo, CaballeroÁvila, \& Rosales-Pérez, 2007).

En Colombia, para el 2017, de acuerdo con información de Forensis, el mayor índice de suicidios se presentó en personas de edades entre los 20 y 39 años (44.73\%). Éste es un proceso que empieza con la ideación suicida y que finaliza con el suicidio (Instituto de
Medicina Legal y Ciencias Forenses, 2018).

El riesgo de suicidio aumenta cuando las ideas y sentimientos alrededor del pensamiento de suicidarse se vuelven constantes y se acompañan de cambios en la conducta o planes en torno al suicidio (Cabra, Infante, \& Sossa, 2010; Rodríguez, García, \& Caliope, 2005).

Múltiples estudios han identificado factores que inciden en la ideación suicida, como el insomnio, la falta de apoyo por parte de la familia, trastornos adaptativos y enfermedades psiquiátricas con trastorno del afecto (depresión mayor, trastorno afectivo bipolar), entre otros (Córdova-Osnaya et al., 2007; González, Díaz, Ortiz, GonzálezForteza, \& González, 2000; Sun \& Hui, 2017; Díaz et al., 2010; Wild, Fisher, \& Lombard, 2004). En estudiantes universitarios aparece como factor de riesgo importante el estrés estudiantil (Wild et al., 2004).

Así mismo, los trastornos adaptativos corresponden a la frecuencia más alta encontrada en el grupo de jóvenes universitarios (Córdova-Osnaya et al., 2007). Es importante destacar que el suicidio es un fenómeno complejo donde actúan tanto factores físicos, como sociales, culturales y psicológicos (Micin \& Bagladi, 2011). Así, el número de factores que pueden conducir a un individuo a construir la idea de cometer una conducta suicida es casi infinito.

Por todo lo anterior, la presente investigación tiene como objetivo identificar la frecuencia del riesgo suicida y sus factores asociados en una universidad de la ciudad de Manizales (Colombia). 


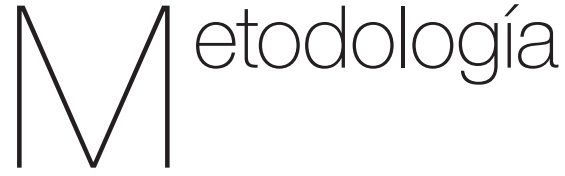

El presente es un estudio cuantitativo de corte trasversal analítico efectuado en una muestra de 170 estudiantes (de una población total de 2,360 matriculados en el $1^{\circ}$ semestre del año 2017) de una universidad de la ciudad de Manizales (Colombia). Los participantes fueron escogidos mediante un muestreo por conveniencia, teniendo en cuenta como criterios de inclusión que cursaran un programa presencial de primero a cuarto semestre y se encontraran entre los 15 a 19 años de edad.

A continuación, se describen las escalas empleadas:

\section{Cuestionario ISO-30}

El cuestionario ISO-30 es un instrumento de tamizaje que consta de 30 ítems que detectan posible presencia de riesgo suicida. Es autoadministrable, los ítems son formulados en forma positiva y negativa (directos e inversos) y se responden en una escala tipo likert de cuatro posiciones ( 0 : totalmente en desacuerdo, 1: en parte en desacuerdo, 2: en parte de acuerdo y 3 : totalmente de acuerdo). Fue diseñado por King y Kowalchuk (1994), y adaptado para ser empleado en adolescentes de Buenos Aires, Argentina (Fernández-Liporace \& Casullo, 2006). Este instrumento ha sido empleado anteriormente en población colombiana (Rodríguez-Escobar, Medina-Pérez, Rozo-David, \& SánchezGarcía, 2013; Rubio et al., 2014). En virtud de su brevedad y sensibilidad, es una herramienta útil para tamizaje. Arroja una puntuación total que permite clasificar al sujeto en tres categorías de riesgo suicida: bajo, moderado y alto.

\section{Cuestionario APGAR}

FAMILIAR

El APGAR familiar es una escala de percepción de cada miembro de la familia del nivel de funcionalidad familiar. Se compone de cinco preguntas que se contestan mediante una escala likert de cinco niveles. Clasifica la función familiar como: buena función familiar, disfunción leve, disfunción moderada y disfunción severa.

Este instrumento ha sido validado en población colombiana en estudiantes de básica secundaria (Forero-Ariza, Avendaño-Durán, Duarte-Cubillos, \& Campo-Arias, 2006) y en pacientes odontológicos colombianos (Díaz-Cárdenas, Tirado-Amador, \& SimancasPallares, 2017).

\section{Inventario de Depresión de Beck}

El inventario de depresión de Beck (Beck, Steer, \& Carbin, 1998) es una de las pruebas utilizadas para evaluar los síntomas depresivos en adolescentes y adultos. Presenta una sensibilidad del $73 \%$ y una especificidad del 68\% (Bonicatto, Dew, \& Soria, 1998). Las categorías que emplea son las siguientes: sin síntomas de depresión o síntomas mínimos, síntomas de depresión leve a moderada, síntomas de depresión moderada a severa y síntomas de depresión grave (Beck et al., 1998). Ha sido validado en población española (Sanz 
\& Vázquez, 1998) y chilena (MelipillánAraneda, Cova-Solar, Rincón-González, \& Valdivia-Peralta, 2008).

\section{Escala de autoestima de Rosenberg}

La Escala de autoestima de Rosenberg mide autoestima, entendida como los sentimientos de valía personal y de respeto a sí mismo. La escala consta de 10 ítems, que se responde en una escala tipo likert: 1 = Totalmente en desacuerdo; 2 = De acuerdo; 3 = En desacuerdo; 4 = Totalmente en desacuerdo, y emplea las siguientes categorías: autoestima elevada, autoestima media y autoestima baja.

Este instrumento ha sido validado en población española (Vázquez-Morejón, García-Boveda, \& Vázquez-MorejónJiménez, 2004); además, en Chile fue traducida y validada en una población de adultos jóvenes y adultos mayores (Rojas-Barahona, Zegers, \& Förster, 2009), así como también ha sido validada en una población de estudiantes de Cartagena (Colombia) pero planteando que la escala no se comporta de forma unidimensional, como se reporta en esta investigación, sino de forma bidimensional (Cogollo, Campo-Arias, \& Herazo, 2015).

\section{Cuestionario Scoff}

El cuestionario Scoff es un instrumento de cinco ítems para el tamizaje de trastornos de la conducta alimentaria. Ha sido validado en adolescentes escolarizados de la ciudad de Bucaramanga (Colombia) (Rueda-Jaimes, Díaz-Martínez, Ortiz-Barajas, PinzónPlata, \& Rodríguez-Martínez, 2005), en adolescentes escolarizados de la misma ciudad (Campo-Arias, Díaz-Martínez, \& Rueda-Jaimes, 2006), además de mujeres universitarias, también en la ciudad de Bucaramanga (Rueda et al., 2005).

\section{Escala AUDIT}

La escala AUDIT es un cuestionario que identifica los trastornos debidos al consumo de alcohol. Tiene una versión chilena (Alvarado, Garmendia, Acuña, Santis, \& Arteaga, 2009) y ha sido validada en población universitaria chilena (Seguel-Palma, Santander-Manríquez, \& Barriga, 2013), así como en estudiantes universitarios colombianos (CampoArias, Villamil-Vargas, \& Herazo, 2013).

\section{Escala de Epworth}

La escala Epworth es un cuestionario corto tipo Likert que mide la somnolencia diurna. Ha sido validada en Colombia con pacientes de centros de sueño en Bogotá, Pereira y Cali (ChicaUrzola, Escobar-Córdoba, \& EslavaSchmalbach, 2007).

\section{Consideraciones éticas}

En esta investigación se respetaron todas las normas éticas de ley vigentes en Colombia para proyectos en Ciencias de la Salud.

\section{Análisis estadístico}

En esta investigación las variables medidas en escala nominal fueron 
desplegadas mediante tablas de frecuencia y límites de confianza al 95\%, mientras que las variables medidas en escala numérica fue mediante promedios, medianas, desviaciones estándar y límites de confianza al 95\%. Referente a la estadística inferencial, las relaciones bivariadas entre variables medidas en escala nominal se determinaron mediante el procedimiento estadístico de $\chi^{2}$. El análisis multivariado se efectuó a través del procedimiento de regresión logística multinomial (todos los análisis de inferencia estadística se hicieron con un $\alpha=.05)$. Los valores faltantes no fueron incluidos en los cálculos y a las escalas empleadas se les calculó la $\alpha$ de Cronbach.

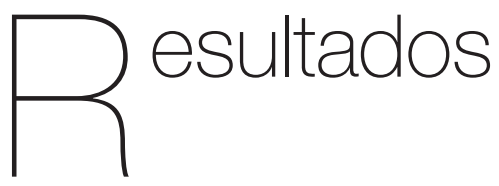

En la investigación participaron 170 estudiantes que cursaban de $1^{\circ}$ a $4^{\circ}$ semestre en una universidad de la ciudad de Manizales (Colombia). Los participantes tenían una edad promedio de 20 años y el 55.9\% ( $n=95)$ pertenecían al género femenino. La Tabla 1 muestra las variables demográficas de esta población, y también el Riesgo Suicida (RS), variable central del estudio, que resultó ser "alto" en el 19.4\% ( $n$ = 33) de la población. También se encuentra el factor de riesgo suicida dicotómico, calculado asignando el valor "sin" al RS "bajo", y el valor "con" (44.7\% $(n=76)$ a los niveles de RS "moderado" y "alto".

Tabla 1

Variables demográficas y factor de riesgo para ideación suicida de la población participante

\begin{tabular}{|c|c|c|c|c|}
\hline Variable & Niveles & N & $\%$ & LC $95 \%$ \\
\hline \multirow{2}{*}{ Género } & Femenino & 95 & 55.9 & $49.4-62.4$ \\
\hline & Masculino & 75 & 44.1 & $37.6-51.2$ \\
\hline \multirow{3}{*}{ Edad (años) } & Promedio & 20 & & $19.5-20.4$ \\
\hline & Mediana & 19 & & \\
\hline & Desviación Estándar & 3 & & \\
\hline \multirow{2}{*}{ Procedencia } & Urbana & 146 & 85.9 & $80.6-90.6$ \\
\hline & Rural & 24 & 14.1 & $9.4-18.8$ \\
\hline \multirow{4}{*}{ Minorías } & LGBTI & 7 & 58.3 & $41.7-75$ \\
\hline & Afro-descendiente & 4 & 33.3 & $16.7-50$ \\
\hline & Indígena & 1 & 8.3 & $0-25$ \\
\hline & Válidos & 12 & 7.1 & \\
\hline \multirow{6}{*}{$\begin{array}{l}\text { Estrato social vivienda } \\
\text { del estudiante }\end{array}$} & 3 & 57 & 33.5 & $26.5-39.4$ \\
\hline & 4 & 48 & 28.2 & 22.4-34.7 \\
\hline & 2 & 25 & 14.7 & $10.6-19.4$ \\
\hline & 6 & 16 & 9.4 & $5.9-13.5$ \\
\hline & 5 & 16 & 9.4 & $5.9-13.5$ \\
\hline & 1 & 8 & 4.7 & $2.4-7.5$ \\
\hline
\end{tabular}




\begin{tabular}{|c|c|c|c|c|}
\hline Variable & Niveles & N & $\%$ & LC 95\% \\
\hline & Soltero & 158 & 92.9 & $89.4-95.9$ \\
\hline \multirow[t]{6}{*}{ Estado civil } & Unión libre & 6 & 3.5 & $1.8-5.9$ \\
\hline & Casado & 6 & 3.5 & $1.8-5.9$ \\
\hline & Derecho & 44 & 25.9 & $20.6-31.8$ \\
\hline & Medicina & 24 & 14.1 & 8.8-19.4 \\
\hline & Contaduría & 19 & 11.2 & $7.6-15.3$ \\
\hline & Ingeniería de Sistemas & 18 & 10.6 & $6.5-14.7$ \\
\hline \multirow[t]{7}{*}{ Programa } & Admon. de Empresas & 17 & 10.0 & $6.5-13.5$ \\
\hline & Psicología & 16 & 9.4 & $5.9-12.9$ \\
\hline & Comunicación Social & 14 & 8.2 & $4.7-11.8$ \\
\hline & Mercadeo & 14 & 8.2 & $4.7-11.8$ \\
\hline & Lic. Inglés & 4 & 2.4 & $.6-4.7$ \\
\hline & 3 & 66 & 38.8 & $32.4-45.9$ \\
\hline & 2 & 38 & 22.4 & $16.5-28.2$ \\
\hline \multirow{2}{*}{ Semestre } & 1 & 38 & 22.4 & $17.1-27.6$ \\
\hline & 4 & 28 & 16.5 & $11.8-21.2$ \\
\hline Riesgo Suicida & Bajo & 94 & 55.3 & $48.8-61.8$ \\
\hline (Cuestionario ISO30) & Moderado & 43 & 25.3 & $19.4-31.8$ \\
\hline$\alpha$ de Cronbach $=.899$ & Alto & 33 & 19.4 & $14.1-25.3$ \\
\hline \multirow{2}{*}{ Riesgo Suicida (dicotómica) } & $\operatorname{Sin}$ & 94 & 55.3 & $48.8-61.2$ \\
\hline & Con & 76 & 44.7 & $37.9-51.8$ \\
\hline
\end{tabular}

Fuente: datos del estudio.

La Tabla 2 evidencia los factores de $\quad 41.8 \%$ acuden a prácticas religiosas, 47.1\% riesgo y protectores para ideación suicida tienen pareja, son de padres separados el presentes en esta población. Según repor- 37.6\%, consumen sustancias psicoactivas taron los estudiantes, se observa que el el 21.8\% y bebidas alcohólicas el 57.6\%.

Tabla 2.

Factores de riesgo y protectores para ideación suicida en la población participante en el estudio.

\begin{tabular}{lllcc}
\hline \multicolumn{1}{c}{ Variable } & \multicolumn{1}{c}{ Niveles } & N & L & LC 95\% \\
\hline \multirow{2}{*}{ Religión } & No & 99 & 58.2 & $51.2-65.9$ \\
& $\mathrm{Si}$ & 71 & 41.8 & $34.7-48.2$ \\
\hline \multirow{3}{*}{ Cuál religión } & Católica & 54 & 76.1 & $66.2-84.5$ \\
& Cristiana & 11 & 15.5 & $8.5-22.5$ \\
& Ofel & 4 & 5.6 & $1.4-11.3$ \\
& Reynum cristi & 1 & 1.4 & $0-4.2$ \\
& Evangélica & 1 & 1.4 & $0-4.2$ \\
& Válidos & 71 & 41.8 & Continúa
\end{tabular}




\begin{tabular}{|c|c|c|c|c|}
\hline Variable & Niveles & N & $\%$ & LC95\% \\
\hline \multirow{2}{*}{ Pareja } & No & 90 & 52.9 & $45.9-60$ \\
\hline & $\mathrm{Si}$ & 80 & 47.1 & $40-54.1$ \\
\hline \multirow{4}{*}{$\begin{array}{l}\text { Cómo es la } \\
\text { relación de pareja }\end{array}$} & Estable & 62 & 77.5 & $68.8-85$ \\
\hline & Abierta & 10 & 12.5 & $7.5-17.5$ \\
\hline & Conflictiva & 8 & 10.0 & $5-15$ \\
\hline & Válidos & 80 & 47.1 & \\
\hline \multirow{2}{*}{ Padres separados } & No & 106 & 62.4 & $54.7-69.4$ \\
\hline & $\mathrm{Si}$ & 64 & 37.6 & $31.2-44.7$ \\
\hline \multirow{2}{*}{ Consumo sustancias } & No & 133 & 78.2 & $72.4-83.5$ \\
\hline & $\mathrm{Si}$ & 37 & 21.8 & $15.9-27.6$ \\
\hline \multirow{6}{*}{ Qué sustancias } & Marihuana & 31 & 83.8 & 73-91.9 \\
\hline & Cocaína & 2 & 5.4 & $0-13.5$ \\
\hline & Marihuana y anfetaminas & 2 & 5.4 & $0-13.5$ \\
\hline & Anfetaminas & 1 & 2.7 & $0-8.1$ \\
\hline & Marihuana y cocaína & 1 & 2.7 & $0-8.1$ \\
\hline & Válidos & 37 & 21.8 & \\
\hline \multirow{4}{*}{$\begin{array}{l}\text { Frecuencia de } \\
\text { consumo (veces por mes) }\end{array}$} & Promedio & 15 & & $9.2-20.6$ \\
\hline & Mediana & 9 & & \\
\hline & Desviación Estándar & 17.1 & & \\
\hline & Válidos & 37 & 21.8 & \\
\hline \multirow{2}{*}{ Consumo de alcohol } & $\mathrm{Si}$ & 98 & 57.6 & $51.2-64.1$ \\
\hline & No & 72 & 42.4 & $35.3-48.8$ \\
\hline \multirow{2}{*}{ Trabaja } & No & 143 & 84.1 & $78.8-89.1$ \\
\hline & $\mathrm{Si}$ & 27 & 15.9 & $11.2-21$ \\
\hline \multirow{7}{*}{ Qué trabajo } & Sistemas & 3 & 11.1 & $0-25.9$ \\
\hline & Auxiliar contable & 2 & 7.4 & $0-18.5$ \\
\hline & Asesor comercial & 2 & 7.4 & $0-18.5$ \\
\hline & Restaurante & 2 & 7.4 & $0-18.5$ \\
\hline & Mesero & 2 & 7.4 & $0-18.5$ \\
\hline & Otros & 16 & 59.2 & \\
\hline & Válidos & 27 & 15.9 & \\
\hline \multirow{2}{*}{ Hijos } & No & 161 & 94.7 & $91.2-97.6$ \\
\hline & $\mathrm{Si}$ & 9 & 5.3 & $2.9-7.6$ \\
\hline \multirow{4}{*}{ Cuantos hijos } & Promedio & 1.8 & & $.94-2.62$ \\
\hline & Mediana & 1 & & \\
\hline & Desviación Estándar & 1.1 & & \\
\hline & Válidos & 8 & 5.3 & \\
\hline
\end{tabular}


Continuación

\begin{tabular}{|c|c|c|c|c|}
\hline Variable & Niveles & $\mathrm{N}$ & $\%$ & LC95\% \\
\hline \multirow{2}{*}{ Personas a cargo } & No & 159 & 93.5 & $90-97.1$ \\
\hline & $\mathrm{Si}$ & 11 & 6.5 & $3.5-8.8$ \\
\hline \multirow{4}{*}{ Cuantas personas a cargo } & Promedio & 2.3 & & $1.23-3.32$ \\
\hline & Mediana & 2 & & \\
\hline & Desviación Estándar & 1.6 & & \\
\hline & Válidos & 11 & 6.5 & \\
\hline
\end{tabular}

Fuente: elaboración propia.

En la Tabla 3 se encuentran otros factores de riesgo para ideación suicida, que provienen de la calificación de las diferentes escalas empleadas en esta investigación. Se destaca que el 24.1\% presenta un consumo riesgoso de bebidas alcohólicas y el 31.2\% tiene autoestima baja.
Se calculó la $\alpha$ de Cronbach (fiabilidad) para cada escala, estando dentro de lo previsto en las diferentes validaciones de los cuestionarios. Lo anterior indica que fueron adecuadamente respondidos por la población de estudio.

Tabla 3.

Resumen de cuestionarios aplicados a la población de estudiantes universitarios.

\begin{tabular}{|c|c|c|c|c|}
\hline Variable & Niveles & $\mathrm{N}$ & $\%$ & LC95\% \\
\hline \multirow{4}{*}{$\begin{array}{l}\text { Funcionalidad familiar } \\
\text { (Apgar familiar) } \alpha \text { de } \\
\text { Cronbach }=.928\end{array}$} & Buena funcionalidad & 105 & 61.8 & $53.8-69.4$ \\
\hline & Disfunción leve & 36 & 21.2 & $15.3-26.5$ \\
\hline & Disfunción moderada & 16 & 9.4 & $5.9-12.9$ \\
\hline & Disfunción severa & 13 & 7.6 & $4.7-10.6$ \\
\hline \multirow{4}{*}{$\begin{array}{l}\text { Depresión (Cuestio- } \\
\text { nario de Beck) } \alpha \text { de } \\
\text { Cronbach }=.921\end{array}$} & $\operatorname{Sin}$ & 126 & 74.1 & $67.6-80.6$ \\
\hline & Leve a moderada & 22 & 12.9 & $8.8-17.1$ \\
\hline & Moderada a severa & 17 & 10.0 & $5.9-14.1$ \\
\hline & Grave & 5 & 2.9 & $.6-5.9$ \\
\hline \multirow{3}{*}{$\begin{array}{l}\text { Autoestima (Cuestio- } \\
\text { nario de Rosenberg) } \alpha \\
\text { de Cronbach }=.797\end{array}$} & Normal & 81 & 47.6 & $40.6-54.1$ \\
\hline & Baja & 53 & 31.2 & $25.3-37.1$ \\
\hline & Alta & 36 & 21.2 & $15.9-27.1$ \\
\hline \multirow{3}{*}{$\begin{array}{l}\text { Dependencia alcohol } \\
\text { (Cuestionario AUDIT) } \\
\alpha \text { de Cronbach }=.895\end{array}$} & No consumo & 72 & 42.4 & $35.9-49.4$ \\
\hline & Consumo no riesgoso & 57 & 33.5 & $27.1-40$ \\
\hline & Con riesgoso & 41 & 24.1 & $18.8-30$ \\
\hline \multirow{4}{*}{$\begin{array}{l}\text { Somnolencia (Cues- } \\
\text { tionario Epworth) } \alpha \\
\text { de Cronbach }=.826\end{array}$} & Ligera & 72 & 42.4 & $35.9-48.8$ \\
\hline & Sin somnolencia & 55 & 32.4 & $25.9-38.2$ \\
\hline & Somnolencia moderada & 32 & 18.8 & $13.5-24.1$ \\
\hline & Somnolencia grave & 11 & 6.5 & $3.5-9.6$ \\
\hline
\end{tabular}


pp • 77-95 José Jaime Castaño Castrillón, Sandra Constanza Cañón Buitrago y Juan José López Tamayo

Continuación

\begin{tabular}{llccc}
\hline \multicolumn{1}{c}{ Variable } & Niveles & N & \% & LC95\% \\
\hline $\begin{array}{l}\text { Trastorno conducta } \\
\text { alimentaria (Cues- }\end{array}$ & Negativo & 134 & 79.3 & $73.4-85.5$ \\
tionario Scoff) $\alpha$ de & Positivo & 35 & 20.7 & $15.4-26$ \\
Cronbach $=.625$ & Válidos & 169 & 99.4 & \\
\hline
\end{tabular}

Fuente: elaboración propia.

\section{Análisis bivariado}

Empleando el procedimiento estadístico de $\chi^{2}$ se analizó la dependencia entre $\mathrm{RS}$ y otros factores de riesgo para ideavariable RS dicotómica, como se define en la Tabla 1. Así, la Tabla 4 presenta las relaciones que resultaron significativas ( $p$ ción suicida. Para este cálculo se tomó la <.05).

Tabla 4.

Relaciones bivariadas significativas ( $p$ <.05) entre FR para ideación suicida (ISO30) y otros factores de riesgo para ideación suicida.

\begin{tabular}{|c|c|c|c|c|}
\hline Variables & Niveles & \multicolumn{2}{|c|}{ Riesgo suicida } & $\mathrm{p}$ \\
\hline & & Con N (\%) & $\operatorname{Sin} N(\%)$ & \multirow{3}{*}{$\begin{array}{c}.020 \\
\text { Rp (f/m): } 2.07 \\
\text { Lc95\%: } 1.12-3.83\end{array}$} \\
\hline \multirow{2}{*}{ Género } & Femenino & $36.8(35)$ & $63.2(60)$ & \\
\hline & Masculino & $54.7(41)$ & $45.3(34)$ & \\
\hline \multirow[b]{2}{*}{ Consumo sustancias } & No & $37.6(50)$ & $62.4(83)$ & \multirow{2}{*}{$\begin{array}{c}.000 \\
\text { Rp (si/no): } 3.92 \\
\text { Lc95\%: } 1.79-8.62\end{array}$} \\
\hline & $\mathrm{Si}$ & $70.3(26)$ & $29.7(11)$ & \\
\hline \multirow{4}{*}{ Funcionalidad familiar } & Buena & $31.4(33)$ & $68.6(72)$ & \multirow{4}{*}{$\begin{array}{c}.000 \\
\text { Rp (mala/buena): } 4.26 \\
\text { Lc95\%: } 2.21-8.24\end{array}$} \\
\hline & Disfunción leve & $61.1(22)$ & $38.9(14)$ & \\
\hline & Disfunción moderada & $68.8(11)$ & $31.3(5)$ & \\
\hline & Disfunción severa & $76.9(10)$ & $23.1(73)$ & \\
\hline \multirow{4}{*}{ Depresión } & Grave & $100(5)$ & $0(0)$ & \multirow{4}{*}{$\begin{array}{c}.000 \\
\text { Rp (con/sin): } 4.97 \\
\text { Lc95\%: } 2.33-10.6\end{array}$} \\
\hline & Leve a Moderada & $63.6(14)$ & $36.4(8)$ & \\
\hline & Moderada a severa & 76.5 (13) & $23.5(4)$ & \\
\hline & $\operatorname{Sin}$ & $34.9(44)$ & $65.1(82)$ & \\
\hline \multirow{3}{*}{ Autoestima } & Alta & $27.8(10)$ & $72.2(26)$ & \multirow{3}{*}{$\begin{array}{c}.000 \\
\text { Rp (anormal/ } \\
\text { normal): } 3.01 \\
\text { Lc95\%: } 1.6-5.65\end{array}$} \\
\hline & Baja & $77.4(41)$ & $22.6(12)$ & \\
\hline & Normal & $30.9(25)$ & $69.1(56)$ & \\
\hline \multirow{3}{*}{ Dependencia alcohol } & Consumo no riesgoso & $31.6(19)$ & $68.4(39)$ & \multirow{3}{*}{$\begin{array}{c}.001 \\
\text { Rp (conrie/conrie): } 3.64 \\
\text { Lc95\%: } 1.72-7.68\end{array}$} \\
\hline & Consumo riesgoso & $68.3(28)$ & $31.7(13)$ & \\
\hline & No consumo & $41.7(30)$ & $58.3(42)$ & \\
\hline \multirow{4}{*}{ Somnolencia } & Grave & $90.9(10)$ & $10.1(1)$ & \multirow{4}{*}{$\begin{array}{c}.003 \\
\text { Rp (tiene/no tiene): } 1.33 \\
\text { Lc95\%: .69.-2.55 }\end{array}$} \\
\hline & Ligera & $36.1(26)$ & $63.9(46)$ & \\
\hline & Moderada & $56.3(18)$ & $43.8(14)$ & \\
\hline & No tiene & $40(22)$ & $60(33)$ & \\
\hline
\end{tabular}

Fuente: elaboración propia 
La Figura 1 muestra la relación entre RS y funcionalidad familiar. Se observa que un $43.42 \%$ de los estudiantes con RS vivencian la funcionalidad familiar de su familia como buena, mientras que aumenta a un $76.6 \%$ entre quienes no presentan RS. La razón de prevalencia calculada como mala funcionalidad/buena funcionalidad, tuvo como resultado un 4.26.

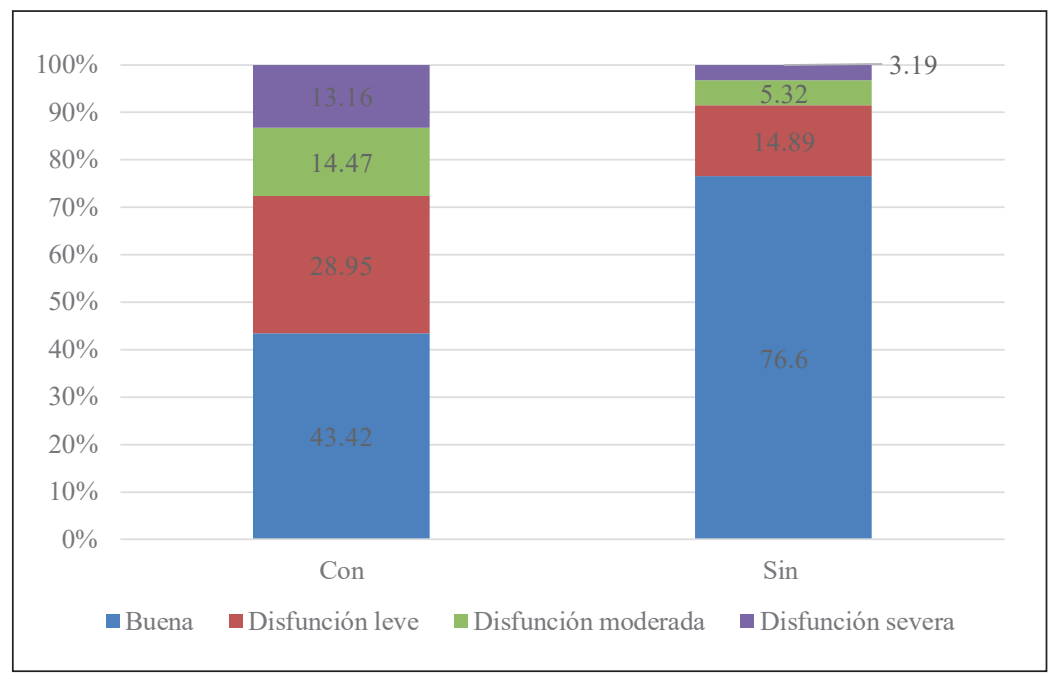

Figura 1. Relación entre riesgo suicida y funcionalidad familiar en estudiantes universitarios de $1^{\circ}$ a $4^{\circ}$ semestre de una universidad de la ciudad de Manizales (Colombia).

Fuente: elaboración propia.

La Figura 2 evidencia la depresión como el factor de riesgo con mayor razón de prevalencia, un RP (con/sin): 4.97; es notorio que el $87.23 \%$ de los participantes sin RS no presentaron niveles de depresión, mientras que esta proporción disminuye a un $57.89 \%$ en los participantes con RS.

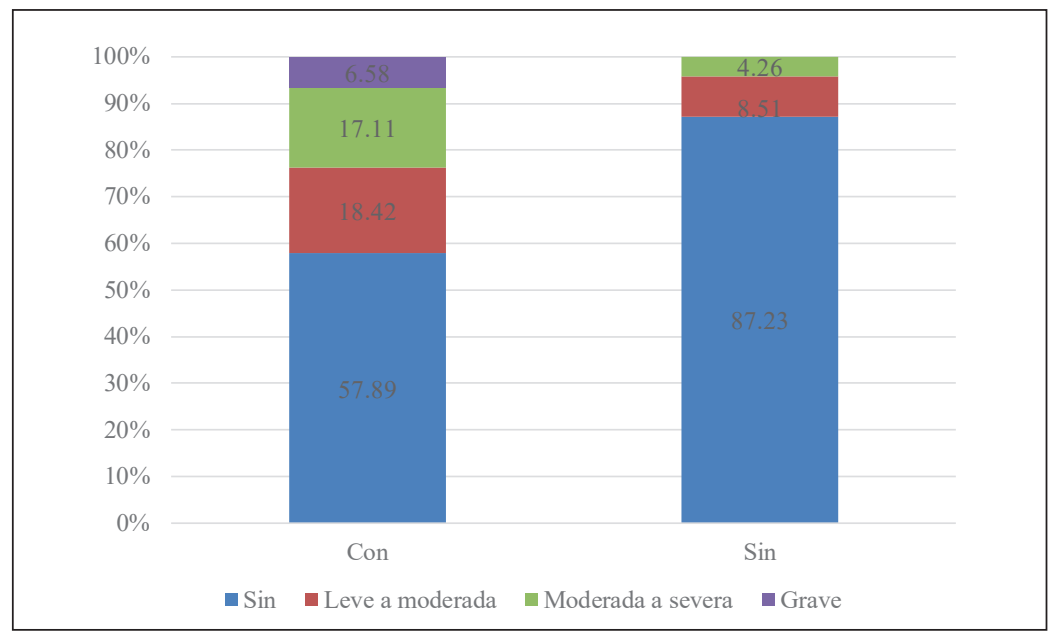

Figura 2. Relación entre riesgo suicida y depresión en estudiantes universitarios de $1^{\circ}$ a $4^{\circ}$ semestre de una universidad de la ciudad de Manizales (Colombia).

Fuente: elaboración propia 


\section{Análisis multivariado}

Para el análisis multivariado se llevó a cabo un análisis de regresión logística multinomial sin término independiente, con $\mathrm{RS}$ como variable dependiente $\mathrm{y}$, como variables independientes, las que resultaron significativas en el análisis bivariado.
La Tabla 5 muestra los resultados de este análisis, hallándose que, en conjunto, el modelo es significativo con $p=.000$.

Los factores de riesgo que resultaron significativos son: funcionalidad familiar ( $p$ $=.022)$, autoestima ( $p=.001)$, y, a un nivel $p<0.1$, somnolencia $(p=.089)$.

Tabla 5.

Modelo multivariado entre riesgo suicida (RS, ISO3O) y los factores de riesgo para ideación suicida.

\begin{tabular}{|c|c|c|c|}
\hline Variable & $\mathrm{X} 2$ & gl & Sig. \\
\hline Modelo & 66.73 & 16 & .000 \\
\hline Género & .044 & 1 & .834 \\
\hline Consumo sustancias & .982 & 1 & .322 \\
\hline Funcionalidad familiar & 9.605 & 3 & .022 \\
\hline Depresión & 3.492 & 3 & .322 \\
\hline Autoestima & 13.475 & 2 & .001 \\
\hline Dependencia alcohol & 1.282 & 2 & .527 \\
\hline Somnolencia & 6.51 & 3 & .089 \\
\hline
\end{tabular}

Fuente: elaboración propia

La Figura 3 ilustra la relación entre RS y los dos factores de riesgo que resultaron significativos en el análisis multivariado. En este sentido, se observa que el nivel "sin" de RS se encuentra, sobre todo, en estudiantes con buena funcionalidad familiar, y autoestima "normal" y "alta".

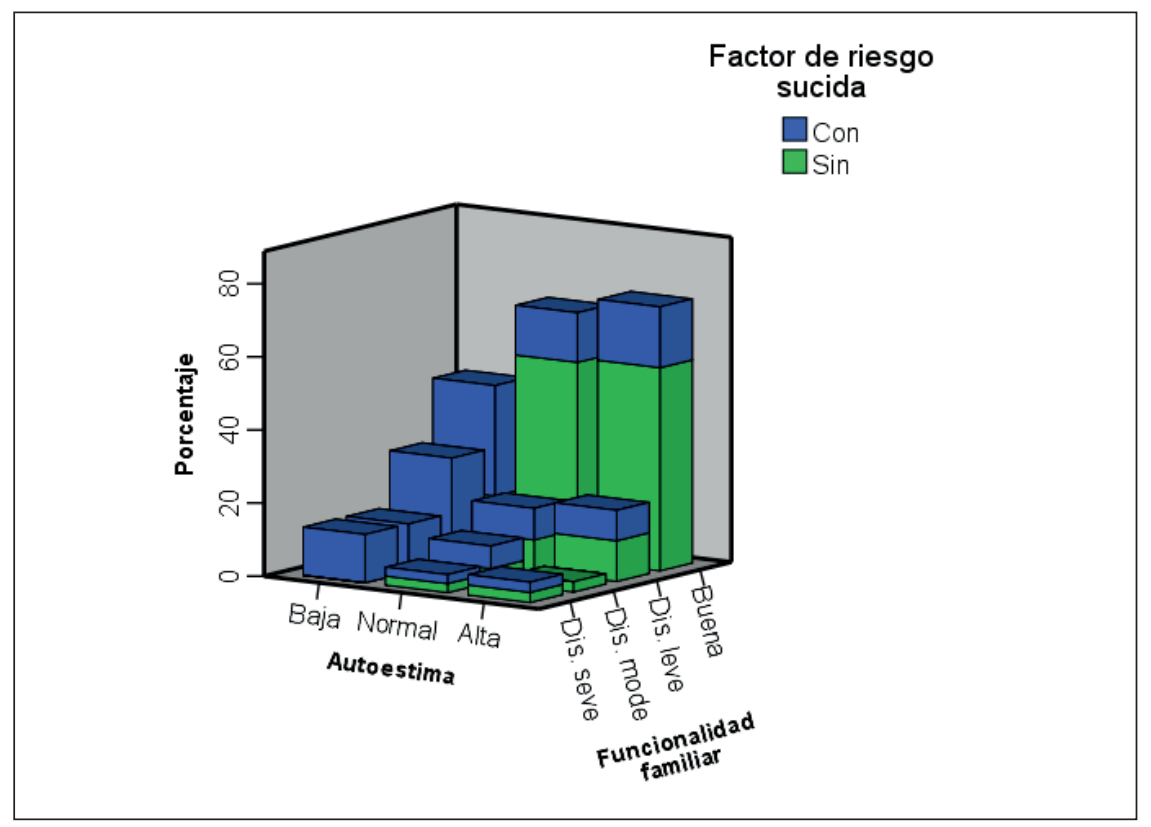

Figura 3. Relación entre RS y los factores de riesgo pata ideación suicida, autoestima y funcionalidad familiar.

Fuente: elaboración propia 


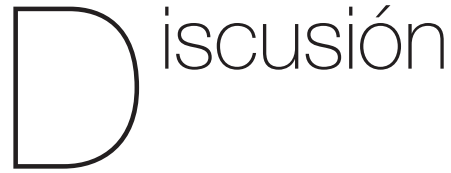

La discusión de resultados trata en primer término de encuadrarlos en un contexto global, tanto en lo referente a la proporción de RS encontrado, como en lo referente a factores asociados significativos en los dos tipos de análisis efectuado. Aparecen en la literatura científica mundial varios artículos que emplean el cuestionario ISO30 para evaluar el riesgo suicida en estudiantes universitarios. Casullo \& Fernández-Liporace ( 2007) encuentraron un RS "alto" que va entre 4.1 y 13.3\% en población argentina semejante. González-Sepúlveda, Medina-Pérez, \& Ortiz-Valencia (2016) hallaron un RS "alto" del 3.6\% en población de estudiantes colombianos. De otro lado, Varengo (2016) encontró en adolescentes escolarizados argentinos un 27.4\% de RS "alto", un 19.5\% "moderado" y un 65.2\% "bajo".

Empleando otras escalas, y en estudiantes universitarios colombianos, Castaño-Castrillón et al. (2015) hallaron un RS de 19.7\%, mientras que Siabato-Macías \& Salamanca-Camargo (2015) un RS de $31 \%$ en población análoga, también en Colombia. En México, Rosales-Pérez, Córdova-Osnaya, y Escobar-Fonseca (2013) detectaron un 7.2\% de ideación suicida, frente a un 8\% hallado por Rosales-Pérez, Córdova-Osuaya, Hernández-Hernández y Zuñiga Villanueva (2014).

Empleando la escala de ideación sucida de Beck, también en México, CórdovaOsnaya et al. (2007) encontraron una proproción de 59.9\% y Sánchez, Cáceres, y Gómez (2002) detectaron una prevalencia de ideación suicida en el momento de la entrevista entre un $4.45 \%$ y un $13 \%$ en estudiantes colombianos.

De todos los estudios mencionados se deduce que el porcentaje "alto" (19.4\%) de la presente investigación se ubica entre los más altos reportados, y semejante al encontrado en otros estudios con población de universitarios colombianos.

Incluyendo factores asociados, BarrosDos Santos, Reschetti-Marcon, MartínezEspinosa, Nunes-Baptista, y Cabral-De Paulo (2017) hallaron un 9.9\% de ideas suicidas y, aplicando el análisis bivariado, el estrato social, orientación sexual, religión, antecedentes familiares de intento suicida, consumo de alcohol y síntomas depresivos presentaron asociación con ideación suicida; en el análisis multivariado aparecen como factores asociados significativos la orientación sexual, antecedentes familiares y los síntomas depresivos.

En el mencionado estudio de CastañoCastrillón et al. (2015) se destacan como factores asociados en el análisis bivariado, el género, preferencias musicales, práctica de deporte, cuadros depresivos y la funcionalidad familiar. Asimismo, SiabatoMacías y Salamanca-Camargo (2015) encontraron como factores asociados el estado civil, eventos vitales estresantes, dependencia emocional, impulsividad y depresión. En este sentido, Rosales-Pérez et al. (2013), en estudiantes mexicanos, identifican como variables asociadas a la ideación suicida, en análisis multivariado, las creencias disfuncionales referidas a la exigencia de éxito, baja tolerancia a la frustración y percepción de apoyo familiar. Además, el estudio de Calvo, Sánchez, y Tejada (2003) muestra en una población de estudiantes colombianos una ideación 
suicida entre el 3.6 y el 18\%, determinando como principales factores relacionados, el antecedente de violación, permanecer más de 8 horas solo y el género femenino.

En estudiantes universitarios de Puerto Rico, una revisión sobre conducta e ideación suicida reporta porcentajes del 12\% (Jiménez-Chafey, 2008). En este estudio se incluyen varias investigaciones efectuadas en Estados Unidos: una, aplicada a 15,977 estudiantes, indicó que el 9.5\% había intentado suicidarse en el último año. Además, una encuesta en 1,455 estudiantes de cuatro universidades, reportó que el 9\% había intentado suicidarse; y, finalmente, en otra investigación con 1,865 estudiantes, el 24\% había pensado en el suicidio, mencionando, como factores de riesgo, síntomas de depresión, desesperanza y estresores académicos, familiares e interpersonales.

En la ya mencionada investigación aplicada a estudiantes universitarios brasileños (Barros-Dos Santos et al., 2017), el análisis bivariado presentó asociación de las ideas suicidas con: clase económica, orientación sexual, práctica religiosa, antecedentes familiares y de amigos, consumo de alcohol y síntomas depresivos; asimismo, en el análisis multivariado deriva asociación con orientación sexual, antecedentes familiares y presencia de síntomas depresivos. Por último, en otro estudio mencionado sobre estudiantes colombianos (Siabato-Macías \& Salamanca-Camargo, 2015) se detectaron como factores asociados el estado civil, unión libre, eventos vitales estresantes, dependencia emocional, impulsividad y depresión.

Según todo lo anterior, existe una amplia gama de factores asociados a los factores de riesgo suicidas (FRS), RS o ideación suicida, aunque cada investigación plantea factores asociados y proporciones diferentes. Un factor que aparece sistemáticamente es la depresión o los síntomas depresivos, que también resultan significativos en esta investigación en el análisis bivariado ( $p=.000, R p$ (con/ sin): 4.97, Lc95\%: 2.33-10.6) (ver Figura 2), más no en el multivariado. Otro factor que aparece con alguna regularidad es la funcionalidad familiar, que en este estudio aparece como significativa tanto en el análisis bivariado ( $\mathrm{p}=.000, \mathrm{Rp}$ (mala) buena): 4.26, Lc95\%: 2.21-8.24) (ver Figura 1), como en el multivariado ( $p=.022$ ). Un factor que en algunas investigaciones es significativo y en otras no, es el género, y que en la presente investigación resultó significativo en el análisis bivariado ( $p$ $=.020, R p(f / m): 2.07$; Lc95\%: 1.12-3.83). Factores estudiados con alguna frecuencia y que también aparecieron como significativos en este estudio, en el análisis bivariado, son el consumo se sustancias psicoactivas ( $\mathrm{p}=.000$, Rp (si/no): 3.92, Lc 95\%: 1.79-8.6) y la dependencia del consumo de alcohol ( $p=.001$, Rp (conrie/connrie): 3.64, Lc95\%: 1.72-7.68). Entre los factores analizados en otras investigaciones y que resultaron significativos, más no en el presente estudio, se destaca la práctica religiosa y el estado civil.

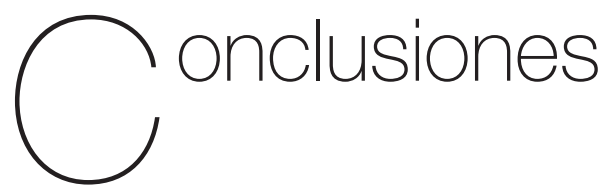

En la presente investigación se detectó un riesgo suicida alto en estudiantes universitarios, similar al nivel de factor de riesgo suicida e ideación suicida encontrada en esta misma población en 
trabajos anteriores. Este RS coincide con el nivel de suicidios en Manizales según el Informe Manizales Cómo Vamos (2017) el cual reporta una tasa de 5.9 suicidios por cada 100,000 habitantes en el año 2016, frente al 5.2/100,000 de Colombia (Instituto de Medicina Legal y Ciencias Forenses, 2018). ¿A qué se debe este alto FRS de estudiantes en Manizales, que desemboca en las más altas tasas de suicidio en Colombia?

En primer lugar, esta población presenta factores asociados al suicidio en alta proporción, como un alto consumo de alcohol (57.6\%) y de sustancias (21.8\%); en esta misma población un estudio anterior mostró un consumo de sustancias psicoactivas de un 29\%, cercano a los límites de confianza de la frecuencia detectada en este estudio (15.9-27.6\%). Presenta también una alta proporción de disfunción familiar (38.2\%) y alto riesgo de depresión, entre otros. Esta alta presencia de factores de riesgo asociados hace que sea urgente actuar sobre esta población para tratar de disminuir el RS alto que se presenta. Estos factores asociados podrían mejorarse con procesos educativos tendientes a disminuir la conducta suicida de estudiantes de colegios de secundaria. Estos procesos estarían orientados a aumentar la autoestima, prevenir el consumo de alcohol y sustancias psicoactivas, disminuir la ansiedad y depresión en estos escolares, etc. Ya antes se ha demostrado la eficacia de estos procesos educativos (Cañón et al., 2018), los cuales deben ser esfuerzos continuos, mediante una política pública que financie estos procesos en todos los colegios de secundaria y todos los semestres.

También se debe considerar que Colombia es uno de los países más violentos del mundo; basta ver los noticieros diarios en la televisión nacional, que suelen dedicar los primeros minutos y gran parte del mismo a difundir hechos violentos de todo tipo como asaltos, robos, homicidios, violaciones y maltrato infantil (Gómez-Giraldo, Hernández-Rodríguez, Gutiérrez-Coba, Arango-Forero \& Franco-Arango, 2010). ¿Es este ambiente de violencia el que estaría influyendo en las ideas suicidas de algunos jóvenes? ¿Depende de nuestra cultura y educación -o por falta de ella- o de nuestra propia naturaleza étnica?

Como toda investigación por encuesta, la presente tiene limitaciones en cuanto a la confiabilidad de los resultados, por las dudas que puedan surgir en cuanto a la honestidad de las respuestas por parte de los estudiantes a los cuestionarios; sin embargo, en este caso, los $\alpha$ de Cronbach obtenidos en los cuestionarios son altos, lo que significa que las respuestas, al ser consistentes, no son al azar. También por ser una investigación de tipo transversal hay una debilidad en cuanto a la determinación de las relaciones causa-efecto. Como se advierte en la metodología del estudio, la muestra escogida es no-probabilística, por lo que los resultados no son generalizables a toda la población de estudio, sin embargo, como se indica en la discusión, el porcentaje de riesgo suicida obtenido está de acuerdo con otros estudios efectuados en esta población.

Conflictos de interés: los autores declaran no tener ningún conflicto de interés.

Fuentes de financiación: Universidad de Manizales. 


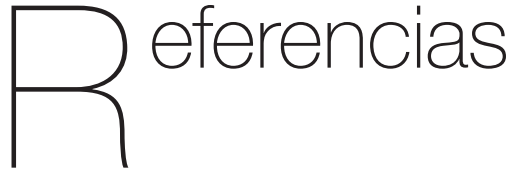

Alvarado, M. E., Garmendia, M. L., Acuña, G., Santis, R., \& Arteaga, O. (2009). Validez y confiabilidad de la versión chilena del Alcohol Use Disorders Identification Test (AUDIT). Revista Médica de Chile, 137, 1463-1468.

Barros-dos-Santos, H. G., Reschetti-Marcon, S., Martínez-Espinosa, M., NunesBaptista, M., \& Cabral de Paulo, P. M. (2017). Factores asociados a la presencia de ideación suicida entre universitarios. Revista Latino-americana de Enfermagem, 25, e2878. http://dx.doi.org/10.1590/15188345.1592.2878.día

Beck, A. T., Steer, R. A., \& Carbin, M. G. (1998). Psychometric properties of the Beck Depression Inventory: Twenty-five years of evaluation. Clinical Psychology Review, 8, 77-100. https://doi. org/10.1016/0272-7358(88)90050-5

Bonicatto, S., Dew, A. M., \& Soria, J. J. (1998). Analysis of the psychometric properties of the Spanish versions of the Beck depression inventory in Argentina. Psychiatry Research, 79, 277-285. https:// doi.org/10.1016/S0165-1781(98)00047-X

Cabra, O., Infante, D., \& Sossa, F. (2010). suicidio y los factores de riesgo asociados en niños y adolescentes. Revista Médica Sanitas, 13(2), 28-35.

Calvo, J., Sánchez, R., \& Tejada, P. (2003). Prevalencia y Factores Asociados a ideación Suicida en Estudiantes Universitarios. Revista de Salud Pública, 5(2), 123-143.
Campo-Arias, A., Díaz-Martínez, L. A., \& Rueda-Jaimes, G. E. (2006). Consistencia interna y análisis factorial del cuestionario scoff para tamizaje de trastorno de conducta alimentaria en adolescentes estudiantes: una comparación por género. Universitas Psychologica, 5(2), 295-304.

Campo-Arias, A., Villamil-Vargas, M., \& Herazo, E. (2013). Confiabilidad y dimensionalidad del Audit en estudiantes de medicina. Psicología desde el Caribe, 28(2), 276-282.

Castaño-Castrillón, J. J., Cañón, S. C., Betancur-Betancur, M., CastellanosSáncherz, P. L., Guerrero-González, J., Gallego-Rivas, A., \& Llanos-Calderón, C. (2015). Factor de riesgo suicida según dos cuestionarios, y factores asociados en estudiantes de la Universidad Nacional de Colombia sede Manizales. Diversitas, 11(2), 193-205.

Cañón, S.C., Castaño-Castrillón, J.J., Mosquera-Lozano, A.M., Nieto-Angarita, L.M., Orozco-Daza, M., \& GiraldoLondoño, W.F. (2018). Propuesta de intervención educativa para la prevención de la conducta suicida en adolescentes en la ciudad de Manizales (Colombia). Diversitas-Perspectivas en Psicología, 14(1), 27-40. http://dx.doi.org/10.15332/ s1794-9998.2018.0001.02

Casullo, M. M., \& Fernández-Liporace, M. (2007). Estudiantes adolescentes argentinos en riesgo suicida: una investigación comparativa. Ciencias Psicológicas, 1(1), 37-45.

Ceballos, G., \& Suárez, Y. (2012). Características de inteligencia emocional y su relación con la ideación suicida en una muestra de estudiantes de Psicología. CES Psicología, 5(2), 88-100. 
Chica-Urzola, H. L., Escobar-Córdoba, F., \& Eslava-Schmalbach, J. (2007). Validación de la escala de somnolencia de Epworth. Revista Salud Pública, 9(4), 558-567.

Cogollo, Z., Campo-Arias, A., \& Herazo, E. (2015). Escala de Rosenberg para autoestima: consistencia interna y dimensionalidad en estudiantes de Cartagena, Colombia. Psychologia: Avances de la Disciplina, 9(2), 61-71.

Córdova-Osnaya, M., Rosales-Murillo, M. D., Caballero-Ávila, R., \& Rosales-Pérez, J. C. (2007). Ideación Suicida en Jóvenes Universitarios: su Asociación con Diversos Aspectos Psicosociodemográficos. Psicología Iberoamericana, 15(2), 17-21.

Díaz-Cárdenas, S., Tirado-Amador, L., \& Simancas-Pallares, M. (2017). Validez de constructo y confiabilidad de la APGAR familiar en pacientes odontológicos adultos de Cartagena, Colombia. Salud UIS, 49(4), 541-548.

Díaz, L., Azevedo, R., Jansen, K., Peretti, R., Lessa, B., \& Pinheiro, R. (2010). Suicidal ideation in adolescents aged 11 to 15 years: prevalence and associated factors. Revista Brasileira de Psiquitria, 32(1), 37-41.

Fernández-Liporace, M., \& Casullo, M. M. (2006). Validación factorial de una escala para evaluar riesgo suicida. Revista Iberoamericana de Diagnóstico y Evaluación e Avaliação Psiocológica RIDEP, 1(21), 9-22.

Forero-Ariza, L. M., Avendaño-Durán, M. C., Duarte-Cubillos, Z. J., \& Campo-Arias, A. (2006). Consistencia interna y análisis de factores de la escala APGAR para evaluar el funcionamiento familiar en estudiantes de básica secundaria. Revista Colombiana de Psiquiatría, 35(1), 23-29.

Gómez-Giraldo, J.C., Hernández-Rodríguez, J.C., Gutiérrez-Coba, L.M., Arango-Forero, G.A., Franco-Arango, A.C. (2010). Los noticieros de la televisión colombiana "en observación”. Palabra Clave, 13(2), 217-250.

González-Sepúlveda, P. C., Medina-Pérez, Ó. A., \& Ortiz-Valencia, J. C. (2016). Riesgo suicida y factores asociados en estudiantes de Psicología en una Universidad pública de Colombia. Revista Habanera de Ciencias Médicas, 15(1), 136-146.

González, S., Díaz, A., Ortiz, S., GonzálezForteza, C., \& González, J. (2000). Características psicométricas de la Escala de Ideación Suicida de Beck (ISB) en estudiantes universitarios de la Ciudad de México. Salud Mental, 23(2), 21-30.

Instituto de Medicina Legal y Ciencias Forenses. (2018). Datos para la vida 2017. Forensis, 19(1), 0-0.

Jiménez-Chafey, M. I. (2008). Conducta e ideación suicida en estudiantes universitarios. Revista Griot, 1(4), 5-17.

King, J., \& Kowalchuk, B. (1994). Manual for ISO-30 Adolescent: Inventory of Suicide Orientation-30. Minneapolis: National Computer Systems, Inc.

Manizales Cómo Vamos (2017). Recuperado de http://manizalescomovamos.org/ en-el-dia-mundial-de-la-salud-suicidiosatencion-de-salud-publica/

Melipillán-Araneda, R., Cova-Solar, F., RincónGonzález, P., \& Valdivia-Peralta, M. (2008). Fiabilidad, validez y datos normativos 
del inventario para la depresión. Terapia Psicológica, 26(1), 59-69.

Micin, S., \& Bagladi, V. (2011). Salud mental en estudiantes universitarios: Incidencia de psicopatología y antecedentes de conducta suicida en población que acude a un servicio de salud estudiantil. Terapia Psicológica, 29(1), 53-64.

Organización Mundial de la Salud. (2019). Suicidio. Recuperado de https://www. who.int/es/news-room/fact-sheets/detail/ suicide

Organización Panamericana de la Salud (2018). Manual de prácticas para el establecimiento y mantenimiento de sistemas de vigilancia de intentos de suicidio y autoagresiones. Recuperado de https://iris.paho.org/bitstream/ handle/10665.2/49120/9789275320082_ spa.pdf?sequence=1\&isAllowed $=y$

Pérez-Olmos, I., Téllez-Cruz, D. L., VélezTraslaviña, Á. L., \& Ibáñez-Pinilla, M. (2012). Caracterización de factores asociados con comportamiento suicida en adolescentes estudiantes de octavo grado, en tres colegios. Revista Colombiana de Psiquiatría, 41(1), 26-46.

Rodríguez-Escobar, A., Medina-Pérez, Ó. A., Rozo-David, A. J., \& Sánchez-García, S. (2013). Ideación suicida y factores asociados en un grupo de policías de Pereira-Colombia. Agora USB, 13(2), 411-420.

Rodríguez, H., García, I., \& Caliope, C. (2005). Resultados de la aplicación dela autopsia psicológica al estudio del suicidio de niños y adolescentes en Uruguay. Revista Medicina de Uruguay, 21, 141-150.
Rojas-Barahona, C., Zegers, B., \& Förster, C. (2009). La escala de autoestima de Rosenberg: Validación para Chile en una muestra de jóvenes adultos, adultos y adultos mayores. Revista Médica de Chile, 137(6), 791-800.

Rosales-Pérez, J. C., Córdova-Osnaya, M., \& Escobar-Fonseca, M. d. (2013). Ideación suicida en estudiantes de la Universidad Tecnológica del Estado de Tlaxcala y variables asociadas. Alternativas en Psicología, 28, 20-32.

Rosales-Pérez, J. C., Córdova-Osuaya, M., Hernández-Hernández, K. E., \& ZuñigaVillanueva, G. (2014). Ideación suicida y variables asociadas en universitarios del altiplano potosino. Psicumex, 4(2), 88-103.

Rubio, L. A., Cardona-Duque, D. V., MedinaPérez, O. A., Garzón-Olivera, L. F., GarzónBorray, H. A., \& Rodríguez-Hernández, N. S. (2014). Riesgo suicida en población carcelaria del Tolima, Colombia. Revista Facultad de Medicina, 62(1), 33-39.

Rueda-Jaimes, G. E., Díaz-Martínez, L. A., Ortiz-Barajas, D. P., Pinzón-Plata, C., \& Rodríguez-Martínez, J. (2005). Validación del cuestionario SCOFF para el cribado de los trastornos del comportamiento alimentario en adolescentes escolarizadas. Atención Primaria, 35(2), 89-94.

Rueda, G. E., Díaz, L. A., Campo-Arias, A., Barros, J. A., Ávila, G. C., Oróstegui, L. T., ... Cadena, L. D. (2005). Validación de la encuesta SCOFF para tamizaje de trastornos de la conducta alimentaria en mujeres universitarias. Biomédica, 25, 196-202.

Sánchez-Sosa, J. C., Villareal-González, M. E., Musitu, G., \& Martínez-Ferrer, B. 
(2010). Ideación Suicida en Adolescentes: Un Análisis Psicosocial. Psychosocial Intervention, 19(3), 279-287.

Sánchez, R., Cáceres, H., \& Gómez, D. (2002). Ideación suicida en adolescentes universitarios: prevalencia y factores asociados. Biomédica, 22, 407-16.

Sanz, J., \& Vázquez, C. (1998). Fiabilidad, validez y datos normativos del inventario para la depresión. Psicothema, 10(2), 303-318.

Seguel-Palma, F., Santander-Manríquez, G., \& Barriga, O. A. (2013). Validez y confiabilidad del test de identificación de los trastornos debidos al consumo de alcohol (audit) en estudiantes de una universidad chilena. Ciencia y Enfermería, 19(1), 23-35.

Siabato-Macías, E. F., \& Salamanca-Camargo, Y. (2015). Factores asociados a ideación suicida en universitarios. Psycholpgia: Avances en la Disciplina, 9(1), 71-81.
Sun, R., \& Hui, E. (2017). Psychosocial factors contributing to adolescent suicidal ideation. Journal Youth Adolescence, 36, 75-86.

Varengo, J. (2016). Ideación suicida en adolescentes (Tesis de Grado). Universidad SIGLO XXI, Córdoba, Argentina.

Vázquez-Morejón, A., Jiménez-García-Boveda, R., \& Vázquez-Morejón-Jiménez, R. (2004). Escala de autoestima de Rosenberg: fiabilidad y validez en población clínica española. Apuntes de Psicología, 22(2), 247-255.

Wild, L., Fisher, A., \& Lombard, C. (2004). Suicidal ideation and attempts in adolescents: association with depression and six domains of self-esteem. Journal of Adolescence, 27, 11-24. 